\title{
Arbeitsmarktinstitutionen und Arbeitslosigkeit
}

Günther Grunert

\begin{abstract}
Trotz des wirtschaftlichen Aufschwungs sind die Forderungen nach einer weiteren Deregulierung des Arbeitsmarktes nicht verstummt. So veröffentlichte die „Initiative Neue Soziale Marktwirtschaft“ im Juli letzten Jahres das Ergebnis einer Umfrage unter 29 „führenden deutschen Wirtschaftswissenschaftlern", nach der eine weitere Flexibilisierung des Arbeitsmarktes die stärksten Impulse für ein dauerhaftes Wirtschaftswachstum bringe und daher die wichtigste politische Maßnahme zur Verstetigung des Aufschwungs sei. Führt eine Arbeitsmarktflexibilisierung tatsächlich zu mehr Wachstum und Beschäftigung? Und sind Arbeitsmarktinstitutionen nichts als Störfaktoren, die Marktunvollkommenheiten und folglich Arbeitslosigkeit verursachen?
\end{abstract}

\section{Einleitung}

In der neoklassischen Modellwelt eines vollständigen und flexiblen Arbeitsmarktes ist der Befund eindeutig: Arbeitslosigkeit - so die Einschätzung - entsteht aus Marktunvollkommenheiten, die der Herausbildung eines markträumenden Gleichgewichtsreallohnsatzes im Wege stehen. Regulierungen des Arbeitsmarktes stellen in dieser Sicht Abweichungen vom reinen Markt dar, erzeugen oder verstärken Marktunvollkommenheiten und führen mithin zu Arbeitslosigkeit. Die Therapie ist ebenso klar: Je stärker alle Regulierungen und Abweichungen vom ansonsten vollkommenen Arbeitsmarkt abgebaut werden, desto mehr wird die Arbeitslosigkeit zurückgehen.

Die Kritik der marktradikalen bzw. neoklassischen Deregulierungsbefürworter richtet sich folglich gegen das ,institutionelle Regelwerk auf dem Arbeitsmarkt" generell und hierbei insbesondere gegen die Kündigungsschutzgesetzgebung ${ }^{1}$, das System der Arbeitslosenunterstützung (die Höhe und Zahlungsdauer der Lohnersatzleistungen) und den Flächentarif. Der Kündigungsschutz führe zu höheren Entlassungskosten und halte damit die Unternehmen von vornherein davon ab, Arbeitskräfte einzustellen; darüber hinaus habe er negative Auswirkungen auf die Flexibilität in der Personalpolitik und damit auf die Produktivität der Betriebe, erhöhe die Kosten der Beschäftigung und mindere so die Einstellungsbereitschaft. Die Zahlung von Lohnersatzleistungen verringere die Arbeitsanreize und Suchaktivitäten der Arbeitslosen; die Folgen seien eine erhöhte Sucharbeitslosigkeit und eine Abschwächung des Wettbewerbs unter den Arbeit- nehmern auf dem Arbeitsmarkt, was den Gewerkschaften die Durchsetzung höherer Löhne ermögliche und so wiederum eine steigende Arbeitslosigkeit verursache. Auch Flächentarifverträge behinderten das freie Spiel von Angebot und Nachfrage auf dem Arbeitsmarkt, führten zu überhöhten Löhnen, einer falschen Lohnstruktur und damit zu höherer Arbeitslosigkeit.

Es ist bereits an anderer Stelle argumentiert worden (Grunert 2004), dass die häufig vertretene These, vom Flächentarifsystem gingen negative Beschäftigungswirkungen aus, weder theoretisch noch empirisch haltbar ist. Im vorliegenden Beitrag soll nun gezeigt werden, dass auch die Behauptung beschäftigungsmindernder Effekte der anderen genannten Arbeitsmarktinstitutionen wenig überzeugend ist, weswegen auch (weitere) Einschränkungen beim Kündigungsschutz und bei der Arbeitslosenunterstützung keineswegs zu einer Verbesserung der Arbeitsmarktsituation führen.

Die Analyse ist wie folgt aufgebaut: Im Abschnitt 2 werden zunächst die Argumente, die die vermeintlich negativen Wirkungen der Kündigungsschutzgesetzgebung und der Arbeitslosenunterstützung (im Folgenden auch vereinfacht als ,institutionelle Schutzsysteme" zusammengefasst) belegen sollen, auf ihre Stichhaltigkeit überprüft. Abschnitt 3 wechselt die Perspektive und fragt danach, ob die genannten Arbeitsmarktinstitutionen Störfaktoren sein können, die einer effizienten Funktionsweise des Arbeitsmarktes entgegenwirken, und welche Auswirkungen auf die Qualifikationsinvestitionen eine Schwächung dieser Institutionen in einem Land wie Deutschland langfristig hat. In Abschnitt 4 steht die in der öffentlichen Diskussion weitgehend ausgeblendete Nachfrageseite im Mittelpunkt; es werden die Folgen eines Abbaus der institutionellen
Schutzsysteme für die gesamtwirtschaftliche Nachfrage analysiert. Abschnitt 5 geht der Frage nach, ob und inwieweit empirische Untersuchungen die herrschende Sicht zu den Arbeitsmarktinstitutionen stützen. Die Analyse endet mit einem Fazit (Abschnitt 6).

\section{Zur Kritik an den Arbeits- marktinstitutionen}

Wie bereits erwähnt, wird oft darauf verwiesen, dass gesetzliche Kündigungsschutzbestimmungen im konjunkturellen Aufschwung zu einer zurückhaltenderen Einstellungspraxis führten. Dieses Argument übersieht jedoch, dass umgekehrt auch im Abschwung die Entlassung von Arbeitskräften erschwert wird. Der Kündigungsschutz verringert folglich die Beschäftigten-Fluktuation (Einstellungen und Entlassungen) und verlängert die durchschnittliche Dauer der Beschäftigungsverhältnisse; der Gesamteffekt auf die Höhe der Arbeits- 
losigkeit bleibt damit theoretisch unbestimmt. Allerdings dürfte sich mit der geringeren Fluktuation der Arbeitskräfte die Struktur der Arbeitslosigkeit ändern. Ein effektiver Kündigungsschutz erhöht die Zahl an stabilen Arbeitsplätzen und sorgt dafür, dass weniger Beschäftigte arbeitslos werden. Arbeitsuchende benötigen jedoch im Durchschnitt länger, um in Beschäftigung (zurück) zu finden. Damit steigt tendenziell die Langzeitarbeitslosigkeit, während die Kurzzeitarbeitslosigkeit sinkt (Nickell/Layard 1999; OECD 1999).

Auch das zweite angeführte Argument gegen die Kündigungsschutzgesetzgebung greift zu kurz: Danach habe der Kündigungsschutz negative Auswirkungen auf die Produktivität, erhöhe die Beschäftigungskosten und senke damit die Einstellungsbereitschaft der Unternehmen, sodass die Zahl der Beschäftigungsverhältnisse abnehme (Bonin 2004, S. 11). Die Ursachen seien, dass unproduktive Beschäftigungsverhältnisse aufrechterhalten würden, der Einsatzwille und die Motivation der Arbeitskräfte aufgrund fehlender Entlassungsdrohungen abnähmen und es den Arbeitnehmern erleichtert werde, sich organisatorischem Wandel zu widersetzen oder unkooperatives Verhalten zu zeigen. Diese Begründung ist einseitig, denn es lässt sich umgekehrt feststellen, dass ein effektiver Kündigungsschutz die Arbeitsplatzsicherheit und Arbeitszufriedenheit erhöht und dadurch die Leistungsbereitschaft der Beschäftigten fördert, die Motivation der Mitarbeiter durch größere Identifikation mit dem Unternehmen verstärkt, über die Stabilisierung der Beschäftigungsverhältnisse zu mehr Loyalität, Vertrauen und Kooperation auf Seiten der Arbeitnehmer führt. Hierdurch werden wesentliche Voraussetzungen für den Einsatz neuer Technologien in den Unternehmen geschaffen. Und insgesamt wird wegen der Verteuerung von Entlassungen eine sorgfältigere Auswahl der Bewerber bei Einstellungen bewirkt, was letztlich auch die Arbeitsproduktivität steigert (zu empirischen Belegen für positive Produktivitätseffekte vgl. Storm/ Naastepad 2007).

Mindestens ebenso wichtig ist ein weiterer Punkt, der kaum Beachtung findet: Die größere Beschäftigten-Fluktuation, die mit einem fehlenden oder schwachen Kündigungsschutz verbunden ist, erhöht nicht nur die Einstellungs-, Einarbeitungs- und Trainingskosten der Unternehmen, sondern verringert auch die Möglichkeiten,
Lerneffekte bei der Produktion zu realisieren: Insbesondere bei neuartigen Produkten mit hohem technologischen Gehalt wachsen im Allgemeinen mit der Zunahme der kumulierten Produktionsmenge (und dem damit verbundenen Ansammeln von Lernerfahrungen) die Kenntnisse und Fertigkeiten der in der Produktion tätigen Arbeitskräfte, was - unabhängig von technologischen Veränderungen - zu bedeutenden Produktivitätssteigerungen (bzw. Stückkostenverringerungen) führt (dazu ausführlicher Grunert 1998, S. 105f.). Der Erwerb eines solchen Erfahrungswissens, das an den einzelnen Betrieb gebunden und nicht einfach auf andere Betriebe übertragbar ist, setzt aber einen bestimmten Grad an Kontinuität voraus, das heißt eine längere Verweildauer kooperierender Beschäftigter (mit gleichem Lernerfahrungsstand) in einem Unternehmen. Mit anderen Worten: „Learning by doing“ ist effektiver, wenn der Personalumschlag in einem Betrieb geringer ist. Da ein stärkerer Kündigungsschutz mit einer niedrigeren Arbeitskräftefluktuation und einer längeren durchschnittlichen Dauer der Betriebszugehörigkeit einhergeht (Storm/Naastepad 2007; Auer et al. 2005), ergibt sich mithin eine positive Wirkung auf den Lernprozess, wodurch Produktivitätswachstum und Verteilungsspielraum erhöht werden.

Was das System der Lohnersatzleistungen anlangt, ist es zwar richtig, dass die Zahlung von Arbeitslosenunterstützung tendenziell die Suchdauer nach einem neuen Arbeitsplatz verlängert. Jedoch ist es verfehlt, jede Verkürzung der Arbeitssuche mit gesamtwirtschaftlichen Wohlfahrtsgewinnen gleichzusetzen. Der Maßstab sollte vielmehr die „optimale Suchdauer" sein, die definiert ist als die „Länge der Arbeitslosigkeitsdauer, die benötigt wird, um einen Beruf auf mindestens gleichem Niveau wie vor Eintritt der Arbeitslosigkeit zu finden, jedoch bevor das Wissen veraltet ist" (Sesselmeier et al. 2006, S. 21). Die Arbeitslosenunterstützung ermöglicht es, dieses Optimum zu erreichen.

Das heißt: Adäquate Lohnersatzleistungen erlauben längere Suchphasen, was zu einem besseren "matching“ zwischen Arbeitsuchenden und offenen Stellen wie auch zu einer längeren Dauer der anschlieBenden Beschäftigungsverhältnisse führt. Dies wiederum geht einher mit positiven Effekten auf das gesamtwirtschaftliche Produktivitätswachstum, den Verteilungsspielraum und die Beschäftigung.
Natürlich ist nicht auszuschließen, dass die optimale Suchdauer überschritten statt unterschritten wird, wenn beispielsweise eine zu ,generöse“ Höhe und/oder Bezugsdauer der Unterstützungszahlungen übermäßig lange Suchphasen auslöst. Jedoch wird diese Gefahr m. E. vielfach überschätzt. Denn die meisten Arbeitslosen wissen, dass sich ihre Wiedereinstellungschancen umso mehr verschlechtern, je länger sie arbeitslos bleiben, da potenzielle Arbeitgeber eine umso geringere Motivation und einen umso höheren Qualifikationsverlust vermuten, je länger ein Bewerber bereits arbeitslos ist. Eine unnötig lange Arbeitslosigkeitsdauer, die zudem das zukünftig zu erwartende Einkommen der Betroffenen reduziert, liegt also nicht im Interesse der Arbeitslosen.

Zusammenfassend lässt sich feststellen, dass die neoklassische Behauptung eindeutig negativer Auswirkungen der Höhe und Zahlungsdauer der Lohnersatzleistungen auf die Suchaktivitäten der Arbeitslosen und damit auf die Beschäftigungssituation zurückgewiesen werden muss. Erwähnt sei noch, dass auch die damit verknüpfte These, die Arbeitslosenunterstützung führe über eine abgemilderte Konkurrenz auf dem Arbeitsmarkt zu höheren Lohnforderungen und entsprechend $\mathrm{zu}$ steigender Arbeitslosigkeit (s. o.), abzulehnen ist, da sie eine strikt inverse Beziehung zwischen Lohn- und Beschäftigungshöhe unterstellt, die sich nicht überzeugend herleiten lässt (dazu ausführlicher Grunert 2003).

\section{Qualifikationen und institutionelle Arrangements}

Institutionelle Regelungen auf dem Arbeitsmarkt werden häufig mit einer „Politik gegen die Märkte“ gleichgesetzt. Tatsächlich aber können soziale Sicherungssysteme helfen, ein Marktversagen im Bereich der Formierung notwendiger Qualifikationen (,skills“) zu überwinden (vgl. zum Folgenden Estevez-Abe et al. 2003; Iversen/Soskice 2001). Die verschiedenen Produktmarktstrategien, die die Unternehmen verfolgen, sind nämlich an die Verfügbarkeit bestimmter Qualifikationen der Beschäftigten gebunden, die wiederum geeignete Formen und Niveaus sozialer Schutzmaßnahmen erfordern. Idealtypisch 
lassen sich drei Arten von Qualifikationen unterscheiden: firmenspezifische, branchenspezifische und allgemeine Qualifikationen. Diese Qualifikationen differieren hinsichtlich ihrer Übertragbarkeit. Firmenspezifische Qualifikationen werden durch Ausbildung am Arbeitsplatz („on-the-job training") erworben und sind selten übertragbar (sie sind wertvoll für das Unternehmen, in dem die Ausbildung stattfindet, aber nicht für andere Unternehmen); branchenspezifische Qualifikationen beziehen sich auf die fachlichen Fertigkeiten und Kenntnisse, die in Berufsausbildung und -schulen vermittelt werden und auf eine bestimmte Branche beschränkt sind; allgemeine Qualifikationen sind betriebs- und branchenunabhängig.

Da der Wert allgemeiner Qualifikationen nicht von einem bestimmten Unternehmen oder einer besonderen Branche abhängt, sind allgemeine Qualifikationen weniger risikoreich als spezifische. Ohne institutionelle Regelungen würde daher ein Arbeitnehmer, der unter der Restriktion unsicherer Erwartungen hinsichtlich seines Arbeitsplatzes handelt, kaum in Qualifikationen investieren, die außerhalb eines bestimmten Unternehmens oder Sektors wenig wert sind, sondern versuchen, allgemeine, übertragbare Qualifikationen zu erwerben, die ihn nicht an einen einzelnen Betrieb oder eine spezielle Branche binden.

Um Investitionen in spezifische Qualifikationen zu ermöglichen und zu fördern, sind institutionelle Arrangements, die Schutz gegen Entlassungen oder Schutz bei Arbeitslosigkeit bieten, unabdingbar: Je höher der Kündigungsschutz, desto geringer ist tendenziell das Entlassungsrisiko, auch in konjunkturellen Abschwüngen, und desto länger ist die durchschnittliche Dauer der Betriebszugehörigkeit; je höher der Arbeitslosigkeitsschutz, desto geringer ist die Einkommensreduktion aufgrund von Arbeitslosigkeit. Sollen Arbeitnehmer in firmenspezifische Qualifikationen investieren, die außerhalb der betreffenden Unternehmen keinen Wert haben, benötigen sie einen hohen Grad an Kündigungsschutz, das heißt eine Garantie, dass sie lange genug in ihren Unternehmen bleiben können, um die Erträge solcher Investitionen (in Form höherer zukünftiger Einkommen) realisieren zu können.

Sind dagegen Investitionen in branchenspezifische Qualifikationen erwünscht, spielt der Kündigungsschutz eine geringere Rolle, da die abhängig Beschäftigten hier im Prinzip ohne Einkommensverluste von der einen zu einer anderen Firma wechseln können (sofern die erforderlichen Qualifikationen rein branchenspezifisch und nicht firmenspezifisch sind). Stattdessen rückt hier der Arbeitslosigkeitsschutz in den Mittelpunkt, das heißt der Schutz der "qualifizierten“ Löhne bzw. der Schutz vor deutlichen Einkommensverringerungen im Fall von Arbeitslosigkeit. Entscheidend sind hier die Quote und die Dauer der Lohnersatzleistungen: Eine hohe Lohnersatzleistungsquote "belohnt" die Arbeitnehmer selbst dann für ihre spezifischen Qualifikationsinvestitionen, wenn sie arbeitslos werden, und beseitigt den Abwärtsdruck auf ihre Löhne, da qualifizierte Arbeitskräfte bei einem Jobverlust nicht zur Annahme von Arbeitsangeboten mit Lohnabschlägen gezwungen sind. Eine längere Zahlungsdauer der Lohnersatzleistungen gibt den arbeitslosen Arbeitnehmern, die in branchenspezifische Qualifikationen investiert haben, genügend Zeit, eine neue Stelle mit "passenden“ Qualifikationsanforderungen (und gleicher Entlohnung wie zuvor) zu finden, insbesondere dann, wenn sie zur Ablehnung von Jobs berechtigt sind, die nicht ihren erworbenen Kernkompetenzen entsprechen.

Wollen Unternehmen in Produktmärkten erfolgreich sein, die in hohem Maße spezifische Qualifikationen erfordern, sind sie auf Beschäftigte angewiesen, die bereit sind, diese Qualifikationen zu erwerben, obgleich dies ihre Abhängigkeit von bestimmten Arbeitgebern oder Arbeitgebergruppen erhöht. Nur bei einer Absicherung gegen die so entstehenden Risiken werden rational handelnde Arbeitnehmer dazu zu bewegen sein.

Institutionelle Regelungen wie Kündigungsschutz und Arbeitslosigkeitsschutz via adäquater Lohnersatzleistungen sind folglich keine Störung der effizienten Funktionsweise von Arbeitsmärkten; vielmehr erhöhen sie die Neigung der Arbeitnehmer, in firmenspezifische respektive branchenspezifische Qualifikationen zu investieren. Dies wiederum reduziert die Kosten der Unternehmen, die ansonsten zur Bereitstellung größerer finanzieller Anreize (z. B. vertraglich vereinbarte hohe Abfindungen bei Entlassungen oder unmittelbare finanzielle Anreize) gezwungen wären - um die Beschäftigten zu motivieren - sich solche für bestimmte Produktmarktstrategien erforderlichen Qualifikationen anzueignen.

Die vorangegangenen theoretischen Überlegungen sind für die Situation in
Deutschland insofern bedeutsam, als sich hierzulande eine große Anzahl von (vor allem größeren) Unternehmen auf eine Kombination von branchenspezifischen und firmenspezifischen Qualifikationen stützt, die zu einem großen Teil im dualen Ausbildungssystem von den Arbeitnehmern erworben werden (im Gegensatz etwa zu den USA oder Großbritannien, wo die dominierenden Produktmarktstrategien der Unternehmen weit mehr auf allgemeinen Qualifikationen, das heißt einer allgemeinen Schul- und Berufsausbildung, basieren). Eine (weitere) Einschränkung des Kündigungs- und des Arbeitslosigkeitsschutzes könnte langfristig durchaus eine abnehmende Bereitschaft der Arbeitnehmer zur Folge haben, Investitionen in die genannten spezifischen Qualifikationen vorzunehmen: Wenn durch die HartzGesetze auch für qualifizierte Arbeitskräfte die Höhe und die Bezugsdauer der Lohnersatzleistungen deutlich gekürzt werden, wenn der früher bestehende Qualifikationsschutz bei der Definition zumutbarer Arbeit gänzlich abgeschafft wird, reduziert dies die Anreize für Arbeitnehmer, branchenspezifische Qualifikationen zu erwerben. Wenn Beschäftigte bei reduziertem Kündigungsschutz schneller entlassen werden können oder nur befristete Arbeitsverträge erhalten, so wird dies kaum ihre Motivation stärken, in firmenspezifische Qualifikationen zu investieren.

\section{Arbeitsmarktinstitutionen und gesamtwirtschaftliche Nachfrage}

Kurzfristig hingegen dürften bei einem Abbau des Kündigungsschutzes und der Leistungen für Arbeitslose die negativen Effekte auf die gesamtwirtschaftliche Nachfrage überwiegen, die aus der Umwandlung bislang kollektiv getragener in individuelle Risiken resultieren (dazu auch Bofinger 2005): Ohne Arbeitslosenversicherung bestünde für jeden Arbeitnehmer das Risiko, bei einem Verlust des Arbeitsplatzes unvermittelt ohne jegliches Einkommen dazustehen; ein Risiko, das er in einen relativ niedrigen, regelmäßigen Beitrag für die Arbeitslosenversicherung und einen erheblich geringeren Einkommensrückgang im Fall eines Arbeitsplatzverlustes transformieren kann. Wird nun von Seiten des 
Staates der Umfang dieser Versicherung eingeschränkt, ist der Einzelne gezwungen, durch eine erhöhte individuelle Vorsorge die aus einem möglichen Jobverlust entstehenden Risiken zu verringern. Er muss mehr sparen, das heißt seine Ausgaben verringern und dabei insbesondere auf den Kauf von Gütern verzichten, die ihn zu festen laufenden Zahlungen über einen längeren Zeitraum hinweg verpflichten, also speziell auf den Erwerb langlebiger und hochwertiger Gebrauchsgüter (z. B. Autos) sowie Immobilien.

Ähnlich ist die Situation beim Kündigungsschutz: Wenn diese kollektive, für alle Unternehmen und ihre Beschäftigten geltende Regelung immer mehr aufgeweicht und damit der Schutz der einzelnen Arbeitnehmer vor willkürlichen Kündigungen reduziert wird, ohne dass das erhöhte Entlassungsrisiko der Beschäftigten durch entsprechende Lohnaufschläge kompensiert wird, bleibt den betroffenen Arbeitnehmern nur die Möglichkeit, weniger zu konsumieren und mehr zu sparen, um für den Fall finanzieller Einbußen bei einem Arbeitsplatzverlust vorzusorgen. Es gilt also für den Arbeitslosigkeits- wie für den Kündigungsschutz: Wird die kollektive Absicherung reduziert, ist der Einzelne zu einer stärkeren individuellen Absicherung gezwungen. Einschränkungen beim Kündigungsschutz und beim Bezug von Arbeitslosenunterstützung erhöhen mithin die ökonomischen Risiken und die Unsicherheit der Arbeitnehmerhaushalte, führen zu höherem Sparen und weniger Konsum, wodurch die gesamtwirtschaftliche Nachfrage geschwächt wird. Gehen die Konsumausgaben zurück, sinkt auch für die Unternehmen der Anreiz, zu investieren. Im Ergebnis wird das Arbeitsmarktproblem nicht gelöst, sondern verschärft.

Der beschriebene Effekt tritt in besonderem Maße in solchen Volkswirtschaften auf, in denen - wie in Deutschland (vgl. Abschnitt 3) - spezifische Qualifikationen eine bedeutende Rolle spielen. Denn erstens ist es für Arbeitskräfte mit diesen Qualifikationen generell schwieriger, nach einem Jobverlust eine geeignete Neuanstellung zu finden. Zweitens aber ist die Dauer der Beschäftigungsverhältnisse im Durchschnitt relativ lang, mit der Folge, dass die Arbeitsmärkte für mittlere Karrierestufen in vielen Beschäftigungskategorien nur schwach entwickelt oder - wie im Extremfall Japan mit seinem (inzwischen unterhöhltem) System der lebenslangen Anstel- lung - in den relevanten Kategorien fast gar nicht existent sind (Carlin/Soskice 2007). Deshalb reagieren die Beschäftigten mit spezifischen Qualifikationen, die in Arbeit verbleiben (und diese bilden die große Mehrheit), auf Arbeitsmarktreformen, die die ökonomische Unsicherheit und die Kosten eines Arbeitsplatzverlustes erhöhen, mit verstärktem Sparen, das heißt dem vorsorglichen Aufbau vermehrter finanzieller Rücklagen.

Zudem ist an einem Abbau der institutionellen Schutzsysteme problematisch, dass - insbesondere durch die Reduzierung der Unterstützungszahlungen an Arbeitslose - ein wesentlicher automatischer Stabilisator der Volkswirtschaft in seiner Wirkung eingeschränkt wird. Automatische Stabilisatoren sind makroökonomische Mechanismen, die konjunkturelle Schwankungen dämpfen, indem sie dafür sorgen, dass sich Nachfrageschocks nur abgeschwächt auf den privaten Konsum auswirken. Eine solche wichtige Rolle kommt auch der Arbeitslosenunterstützung zu, die es denjenigen, die arbeitslos geworden sind, ermöglicht, weiterhin zu konsumieren. Dabei gilt: Je höher das Verhältnis der Ausgaben der Arbeitslosen zum Einkommen der beschäftigten Arbeitnehmer, desto weniger sinkt die Nachfrage, wenn in einem konjunkturellen Abschwung die Arbeitslosigkeit steigt (Robinson 1969, S. 267).

\section{Zur Empirie}

Wie sieht es nun mit den empirischen Belegen für die behaupteten negativen Effekte der institutionellen Regelungen auf die Beschäftigung aus? Seit Beginn der 1990er Jahre ist eine rasch wachsende Zahl von Studien entstanden, in denen der Zusammenhang zwischen Arbeitsmarktinstitutionen und Arbeitslosigkeit im internationalen Vergleich geprüft wird. Diese empirischen Untersuchungen verwenden häufig komplexe multivariate Modelle (oft Paneldatenregressionen), die die Bedeutung einzelner institutioneller Variablen unter Kontrolle einer Vielzahl weiterer Faktoren aufzudecken versuchen. Die einfachste Vorgehensweise ist dabei, für die OECD-Länder die Cross-Country-Beziehung zwischen Arbeitsmarktinstitutionen und Arbeitslosenquoten zu analysieren (Nickell 1997). Eine andere Untersuchungsmethode, die maßgeblich von Blanchard/Wolfers (2000) entwickelt wurde, geht von der Beobachtung aus, dass zwar die Arbeitsmarktinstitutionen in den 1970er Jahren weniger „beschäftigungsfreundlich" geworden seien, die Entwicklung seitdem jedoch eher in die entgegengesetzte Richtung laufe und trotzdem die Arbeitslosigkeit in vielen Ländern weiter anstieg. Die Erklärung für dieses Phänomen vermuten Blanchard und Wolfers in der Interaktion zwischen verschiedenen (stabilen) Institutionen und makroökonomischen Schocks, denen die Länder ausgesetzt waren (Bertola et al. 2001; Fitoussi et al. 2000). Danach erzeugten Arbeitsmarktinstitutionen höhere Arbeitslosigkeit, indem sie die Fähigkeit der Arbeitsmärkte einschränkten, auf adverse Schocks $\mathrm{zu}$ reagieren. Die jeweiligen institutionellen Gegebenheiten bestimmten demnach die Wirkungsstärke dieser Schocks, das heißt die Institutionen in einigen Ländern hätten adverse Schocks in größere Arbeitsmarktprobleme übertragen als diejenigen in anderen Ländern. Ein weiterer Erklärungsversuch der internationalen Unterschiede im Niveau und in der Entwicklung der Arbeitslosigkeit betont die Bedeutung der Interaktionen zwischen den Arbeitsmarktinstitutionen (Belot/van Ours 2001, 2004), während eine wiederum andere Vorgehensweise die Analyse auf die Verwendung jährlicher Daten (statt Mehrjahreszeiträumen) verlagert, um die Veränderung der Arbeitslosenquoten durch zeitvariierende Institutionen-Variablen zu erklären, wobei die Untersuchung bis in das Jahr 1961 zurückgeführt wird (Nickell et al. 2003, 2005). Die Frage der Auswirkungen von Veränderungen bei den Institutionen auf Veränderungen der Arbeitslosigkeit steht auch im Zentrum einer Studie des Internationalen Währungsfonds, die auf einer Vorstudie von Nickell et al. aufbaut, aber einige wesentliche Unterschiede in der Spezifikation aufweist (IMF 2003).

Auffällig ist an all diesen Untersuchungen, die hier nicht im Einzelnen vorgestellt werden können, dass die geschätzten Koeffizienten der Institutionen-Variablen ${ }^{2}$ kei-

\footnotetext{
2 Die am häufigsten verwendeten Variablen sind dabei neben der Quote und der Dauer der Lohnersatzleistungen und dem Kündigungsschutz die aktive Arbeitsmarktpolitik, die Gewerkschaftsdichte, der Deckungsgrad der Tarifverhandlungen, der Koordinationsgrad der Lohnverhandlungen und die Steuern.
} 
neswegs immer statistisch signifikant sind und selbst dort, wo sie signifikant sind, zwischen den verschiedenen Studien erheblich differieren. Die Ergebnisse scheinen mithin wenig robust zu sein; ein Problem, mit dem sich auch Baker, Glyn, Howell und Schmitt in einer fundierten Kritik der empirischen Analysen zum Einfluss der Arbeitsmarktinstitutionen auf die Arbeitslosigkeit auseinandersetzen (Baker et al. 2004, 2005; Howell et al. 2007). Baker et al. zweifeln die Datenqualität der in vielen dieser Untersuchungen eingesetzten institutionellen Variablen an und zeigen am Beispiel der einflussreichen Studien Nickells (1997) und des IWF (IMF 2003), dass bereits kleinere Veränderungen bei den in diesen beiden Arbeiten verwendeten Variablen und sinnvolle Änderungen in der Spezifikation zu Regressionsergebnissen führen, die keinen systematischen Zusammenhang zwischen Arbeitsmarktinstitutionen und Arbeitslosigkeit erkennen lassen (Baker et al. 2005, 2004; Hein et al. 2003).

Aber auch wenn man vom Problem der geringen Robustheit der empirischen Ergebnisse einmal absieht, bleiben erhebliche Zweifel an der Argumentation der Deregulierungs-Befürworter. So gibt es eine Vielzahl ökonometrischer Studien - und darunter gerade jüngere Untersuchungen, die auf neu verfügbaren und verbesserten institutionellen Variablen basieren -, die keinen signifikanten Einfluss der Stärke des Kündigungsschutzes auf die Arbeitslosenquote feststellen oder sogar eine signifikant negative Beziehung zwischen den beiden Variablen ermitteln (Amable et al. 2006; Baccaro/Rei 2005; BAK Basel Economics et al. 2007; Baker et al. 2004, 2005; Bassanini/ Duval 2006; Belot/van Ours 2001, 2004; Daveri/Tabellini 2000; Nickell et al. 2005; Nickell/Layard 1999; OECD 1999, 2006). Selbst der „Sachverständigenrat zur Begutachtung der gesamtwirtschaftlichen Entwicklung" sieht sich in seinem Jahresgutachten 2005/2006 nach einer Auswertung bedeutsamer Studien genötigt einzuräumen, dass sich „(f)ür den Kündigungsschutz ... kein robuster Effekt auf die Höhe der Arbeitslosigkeit belegen (lässt)." (SVR 2005, S. 171).

Was die Quote und die Dauer der Lohnersatzleistungen betrifft, scheint die Empirie die herrschende Theorie schon eher zu stützen: Tatsächlich weisen beide Variablen in einer Reihe von Untersuchungen einen signifikant positiven Zusammenhang mit der Arbeitslosigkeit auf (vgl. z. B. die Lite- raturübersicht in OECD 2006, S. 61, Tab. 3.3), wenngleich es auch hier Studien mit abweichenden Ergebnissen gibt (Baccaro/Rei 2005; BAK Basel Economics et al. 2007; Baker et al. 2004, 2005; Di Tella/MacCulloch 2005; Palley 2006). Allerdings ist das Kausalitätsverhältnis nicht klar: Führt tatsächlich eine zu „großzügige“ Arbeitslosenunterstützung zu mehr Arbeitslosigkeit, oder sind umgekehrt höhere Lohnersatzleistungen die Reaktion der Politik auf eine (längerfristig) gestiegene Arbeitslosigkeit? ${ }^{3}$ Howell et al. (2007) gehen dieser Frage mit Granger-Kausalitätstests ${ }^{4}$ für wichtige einzelne OECD-Länder jeweils im Zeitraum 1962-2004 nach, wobei ihr Hauptaugenmerk auf vier am Arbeitsmarkt zuletzt sehr erfolgreichen europäischen Ländern (Dänemark, Niederlande, Irland, Großbritannien) und vier in dieser Hinsicht wenig erfolgreichen Staaten (Frankreich, Deutschland, Spanien, Italien) liegt. Da in neueren empirischen Studien die Brutto-Lohnersatzleistungsquote der mit Abstand am häufigsten herangezogene Indikator für die "Großzügigkeit" der Arbeitslosenunterstützung ist, der zudem in relativ vielen Untersuchungen einen signifikant positiven Zusammenhang mit der Arbeitslosigkeit aufweist, verwenden ihn auch Howell et al. in ihrer empirischen Analyse. Die Ergebnisse für die vier genannten „Erfolgsländer" sind eindeutig: Wenn ein Zusammenhang zwischen beiden Variablen besteht, dominiert in den vier Ländern (in verschiedenen Tests mit unterschiedlichen Lag-Längen) eindeutig die Wirkungsrichtung von der Arbeitslosigkeit zur Lohnersatzleistungsquote, das heißt die Arbeitslosenrate ist Granger-kausal für die BruttoLohnersatzleistungsquote - und nicht umgekehrt, wie nach der orthodoxen Sichtweise zu erwarten wäre. Was die vier „wenig erfolgreichen" Länder betrifft, so lässt sich für Frankreich und Italien ebenso Granger-Kausalität von der Arbeitslosenrate zur Brutto-Lohnersatzleistungsquote feststellen. Für Deutschland und Spanien trifft dies nicht zu, jedoch ist hier auch umgekehrt die Lohnersatzleistungsquote nicht Granger-kausal für die Arbeitslosenrate, das heißt die Lohnersatzleistungsquote vermag die Arbeitslosigkeit nicht signifikant zu erklären.

Wenngleich eingeräumt werden muss, dass das Konzept der Granger-Kausalität kein „hartes“ Konzept ist, da es zwar eine notwendige, nicht aber eine hinreichende Bedingung für Kausalität analysiert, erge- ben sich dennoch mit der empirischen Untersuchung von Howell et al. (2007) erhebliche Zweifel an der These, dass ein hohes Niveau der Lohnersatzleistungen ursächlich für eine hohe Arbeitslosigkeit ist.

Dagegen wird die in diesem Aufsatz vertretene These eines günstigen Einflusses der Arbeitslosenunterstützung auf den „matching“-Prozess am Arbeitsmarkt und die anschließende Beschäftigungsdauer durch mehrere empirische Studien gestützt (z. B. Centeno 2004; Centeno/Novo 2006; Gangl 2002; Mavromaras 1992). Interessant ist insbesondere die umfangreiche empirische Untersuchung von Gangl (2002) für die USA und Deutschland auf der Basis des U. S. Survey of Income and Program Participation (SIPP) und des Sozio-ökonomischen Panels des DIW (SOEP). Gangls Analyse umfasst für den Zeitraum 19841995 Stichproben von 24.100 Arbeitslosigkeitsperioden von 21.551 Arbeitnehmern im Falle der kombinierten SIPP-Daten und 3.251 Arbeitslosigkeitsperioden von 2.264 Arbeitnehmern beim SOEP (Arbeitnehmer mit und ohne Lohnersatzleistungen). Gangl ermittelt in seiner Studie deutliche positive Effekte der Arbeitslosenversicherung auf die Löhne nach Wiederbeschäftigung. Er kommt aufgrund seiner empirischen Resultate zu dem Schluss, dass die Arbeitslosenversicherung wie eine Suchsubvention für arbeitslose Arbeitnehmer wirke, die auf Kosten einer leichten Verlängerung der Arbeitslosigkeitsdauer zu besseren Ergebnissen bei der Jobsuche, das heißt zu einer erhöhten Qualität der von Arbeitnehmern nach Arbeitslosigkeitsphasen an-

\footnotetext{
3 Bedenkt man, dass beispielsweise in den USA die Verlängerung der Arbeitslosenunterstützung in Zeiten hoher Arbeitslosigkeit fast schon zur gängigen Praxis geworden ist (Blinder 2004, S. 33f.), so kann es nur verwundern, dass die Möglichkeit einer umgekehrten Kausalität (das heißt einer Anhebung der Höhe und Dauer der Unterstützungszahlungen als Reaktion auf eine Zunahme der Arbeitslosigkeit) in nur sehr wenigen Studien in Betracht gezogen wird.

4 Die zugrundeliegende Idee des Granger-Kausalitätstests ist einfach: Wenn $X$ tatsächlich $Y$ verursacht, müssen Veränderungen in $X$ Veränderungen in $Y$ vorausgehen. Zwei Bedingungen müssen insbesondere erfüllt sein, damit man folgern kann, $X$ verursacht $Y$ : Erstens sollte $X$ helfen, $Y$ vorher zusagen, das heißt in einer Regression von $Y$ auf verzögerte Werte von $Y$ müsste die Hinzunahme verzögerter Werte von $X$ als unabhängige Variable signifikant zur Erklärungskraft der Regression beitragen. Zweitens sollte $Y$ nicht helfen, $X$ vorherzusagen. Zur Definition und zum Testverfahren vgl. ausführlich: Pindyck/Rubinfeld 1991, S. $216 f f$.
} 
genommenen Arbeitsstellen und deshalb zu einem verbesserten „matching“ von Bewerbern und offenen Stellen führe. Die Arbeitslosenversicherung trägt also zu einer ausbildungsadäquateren Beschäftigung bei und ist nach Gangl ,eine wichtige institutionelle Einrichtung, um das akkumulierte Humankapital der Arbeitnehmer über Arbeitslosigkeitsperioden hinweg zu erhalten" (Gangl 2002, S. 21; Übersetzung G. G.).

\section{Fazit}

Bedenkt man die dürftige empirische Evidenz für den unterstellten Zusammenhang zwischen dem Grad der Arbeitsmarktregulierung (Kündigungsschutz, Arbeitslosenunterstützung) und der Arbeitslosigkeit, so kann die Vehemenz, mit der Wissenschaft, Politik und Medien in den letzten Jahren für eine Deregulierung des Arbeitsmarktes eingetreten sind, nur erstaunen (Freeman 2008, S. 20-22). Ebenso unverständlich ist es, wenn nun die Abnahme der Arbeitslosigkeit in Deutschland seit dem Frühjahr 2005, die maßgeblich dem Wirtschaftswachstum im gegenwärtigen Aufschwung (der wesentlich von der Exportnachfrage getragen wird) zu verdanken ist, den Arbeitsmarktreformen unter der rot-grünen Regierung zugeschrieben wird. Empirisch belegen lässt sich diese These jedenfalls nicht: So findet Bofinger in einem Vergleich der Aufschwungphase 2005 bis 2007 mit der von 1998 bis 2000 keine Hinweise auf positive Effekte der Arbeitsmarktreformen; die bessere Arbeitsmarktbilanz in der momentanen Aufschwungphase lässt sich demnach primär mit dem schwächeren Anstieg des Arbeitskräfteangebots erklä- ren, bei fast identischer Veränderung der Arbeitskräftenachfrage in beiden Zeiträumen (SVR 2007, S. 341-343, Minderheitsmeinung Bofingers).

Gegenwärtig ist aufgrund der günstigen gesamtwirtschaftlichen Entwicklung in Deutschland die Debatte um die angeblich verkrusteten Arbeitsmärkte etwas abgeflaut, und der Bundestag hat jüngst sogar eine längere Bezugsdauer des Arbeitslosengelds I für ältere Arbeitslose beschlossen. Jedoch kann davon ausgegangen werden, dass spätestens im nächsten konjunkturellen Abschwung wieder verstärkt Forderungen nach radikalen Arbeitsmarktreformen (z. B. einer noch stärkeren Lockerung des Kündigungsschutzes) laut werden. Eine weitere Deregulierung des Arbeitsmarktes würde dann aber nicht nur die Position der abhängig Beschäftigten erneut schwächen, sondern auch eine wirtschaftliche Belebung eher behindern als fördern.

\section{LITERATUR}

Amable, B./Demmou, L./Gatti, D. (2006): Institutions, Unemployment and Inactivity in the OECD Countries, Paris-Jourdan Sciences Economiques, Working Paper 2006-16

Auer, P./Berg, J./Coulibaly, I. (2005): Is a Stable Workforce Good for Productivity?, in: International Labour Review 3, S. 319-343

Baccaro, L./Rei, D. (2005): Institutional Determinants of Unemployment in OECD Countries: A Time Series Cross-Section Analysis (1960-98), DP/160/2005, International Institute for Labour Studies Geneva BAK Basel Economics/IAW Tübingen/Prognos AG Basel (2007): Makroökonomische Flankierung struktureller Reformen im Rahmen der Lissabon-Strategie, Basel

Baker, D./Glyn, A./Howell, D./Schmitt, J. (2004): Unemployment and Labor Market Institutions: The Failure of the Empirical Case for Deregulation www.newschool.edu/cepa/publications/workingpapers/archive/ cepa200404.pdf

Baker, D./Glyn, A./Howell, D./Schmitt, J. (2005): Labor Market Institutions and Unemployment: A Critical Assessment of the Cross-Country Evidence, in: Howell, D. R. (Hrsg.): Fighting Unemployment: The Limits of Free Market Orthodoxy, Oxford, S. 72-118

Bassanini, A./Duval, R. (2006): Employment Patterns in OECD Countries: Reassessing the Role of Policies and Institutions, in: Social, Employment and Migration Working Papers 35

Belot, M./van Ours, J. C. (2001): Unemployment and Labor Market Institutions: An Empirical Analysis, in: Journal of the Japanese and International Economies 4, S. 403-418
Belot, M./van Ours, J. C. (2004): Does the Recent Success of Some OECD Countries in Lowering their Unemployment Rates Lie in the Clever Design of their Labour Market Reforms?, in: Oxford Economic Papers 4, S. 621-642

Bertola, G./Blau, F. D./Kahn, L. M. (2001): Comparative Analysis of Labor Market Outcomes: Lessons for the US from International Long-Run Evidence, in: NBER Working Paper 8526

Blanchard, O./Wolfers, J. (2000): The Role of Shocks and Institutions in the Rise of European Unemployment: The Aggregate Evidence, in: Economic Journal 462, S. C1-C33

Blinder, A. S. (2004): The Case Against the Case Against Discretionary Fiscal Policy, in: CEPS Working Paper 100, June

Bofinger, P. (2005): Wir sind besser, als wir glauben - Wohlstand für alle, München

Bonin, H. (2004): Lockerung des Kündigungsschutzes: Ein Weg zu mehr Beschäftigung?, in: IZA Discussion Paper 1106, April

Carlin, W./Soskice, D. (2007): Reforms, Macroeconomic Policy and Economic Performance in Germany, in: Centre for Economic Policy Research, Discussion Paper 6415

Centeno, M. (2004): The Match Quality Gains from Unemployment Insurance, in: The Journal of Human Resources 3, S. 839-863

Centeno, M./Novo, A. A. (2006): The Impact of Unemployment Insurance on the Job Match Quality: A Quantile Regression Approach, in: Empirical Economics 4, S. 905-919 
Daveri, F./Tabellini, G. (2000): Unemployment, Growth and Taxation in Industrial Countries, in: Economic Policy 30, S. 47-104

Di Tella, R./MacCulloch, R. (2005): The Consequences of Labor Market Flexibility: Panel Evidence Based on Survey Data, in: European Economic Review 5, S. 1225-1259

Estevez-Abe, M./Iversen, T./Soskice, D. (2003): Social Protection and the Formation of Skills: A Reinterpretation of the Welfare State, in: Hall, P.

A./Soskice, D. (Hrsg.): Varieties of Capitalism - The Institutional Foundations of Comparative Advantage, Oxford, S. 145-183

Fitoussi, J.-P./Jestaz, D./Phelps, E. S./Zoega, G. (2000): Roots of the Recent Recoveries: Labor Reforms or Private Sector Forces?, in: Brookings Papers on Economic Activity 1, S. 237-291

Freeman, R. B. (2008): Labor Market Institutions Around the World, in: CEP Discussion Paper 844

Gangl, M. (2002): Unemployment Benefits as a Search Subsidy: New Evidence on Duration and Wage Effects of Unemployment Insurance, Discussion Paper FS I 02-208, Wissenschaftszentrum Berlin für Sozialforschung

Grunert, G. (1998): Technologische Neuerungen und Internationalisierung der Produktion, in: Postler, F./Baron, A. (Hrsg.): Medienforschung, Informationsgesellschaft und ökonomische Entwicklungen - eine kleine Festschrift zum 60. Geburtstag von Herrn Professor Dr. Bernd-Peter Lange am 8. Dezember 1998, Erkrath, S. 97-118

Grunert, G. (2003): Lohnniveau und Beschäftigung, in: WSI-Mitteilungen 6, S. 353-359

Grunert, G. (2004): Der Flächentarif in der Kritik, in: WSI-Mitteilungen 11, S. 596-602

Hein, E./Mülhaupt, B./Truger, A. (2003): WSI-Standortbericht 2003: Standort Deutschland - Reif für radikale Reformen?, in: WSI-Mitteilungen 6, S. 331-343

Howell, D. R./Baker, D./Glyn, A./Schmitt, J. (2007): Are Protective Labor Market Institutions at the Root of Unemployment? A Critical Review of the Evidence, Revised March 2007, in: Capitalism and Society 1, www. newschool.edu/cepa/publications/workingpapers/Howell\%20et\%20al_ Institutions\%20and\%20Unemployment_march15.07.pdf International Monetary Fund (IMF) (2003): Unemployment and Labor Market Institutions: Why Reforms Pay Off, in: World Economic Outlook, April, Washington, S. 129-150

Iversen, T./Soskice, D. (2001): An Asset Theory of Social Policy Preferences, in: American Political Science Review 4, S. 875-893
Mavromaras, K. G. (1992): Zur Arbeitslosigkeits- und Beschäftigungsdauer, in: Mitteilungen aus der Arbeitsmarkt- und Berufsforschung 1, S. 84-90

Nickell, S. (1997): Unemployment and Labor Market Rigidities: Europe versus North America, in: Journal of Economic Perspectives 3, S. 55-74 Nickell, S./Layard, R. (1999): Labor Market Institutions and Economic Performance, in: Ashenfelter, O./Card, D. (Hrsg.): Handbook of Labor Economics, Amsterdam

Nickell, S./Nunziata, L./Ochel, W./Quintini, G. (2003): The Beveridge Curve, Unemployment and Wages in the OECD from the 1960s to the 1990s, in: Aghion, P./Frydman, R./Stiglitz, J./Woodford, M. (Hrsg.): Knowledge, Information, and Expectations in Modern Macroeconomics, Princeton, S. 394-431

Nickell, S./Nunziata, L./Ochel, W. (2005): Unemployment in the OECD Since the 1960s. What Do We Know?, in: Economic Journal 500, S. 1-27 OECD (1999): Employment Outlook, Paris

OECD (2006): Employment Outlook, Paris

Palley, T. I. (2006): The Causes of High Unemployment: Labor Market Sclerosis versus Macroeconomic Policy, in: Hein, E./Heise, A./Truger, A. (Hrsg.): Wages, Employment, Distribution and Growth - International Perspectives, Basingstoke, S. 20-48

Pindyck, R. S./Rubinfeld, D. L. (1991): Econometric Models and Economic Forecasts, New York

Robinson, J. (1969): The Accumulation of Capital, Third Edition, London Sachverständigenrat zur Begutachtung der gesamtwirtschaftlichen Entwicklung (SVR) (2005): Jahresgutachten 2005/2006: Die Chance nutzen - Reformen mutig voranbringen, Stuttgart

Sachverständigenrat zur Begutachtung der gesamtwirtschaftlichen Entwicklung (SVR) (2007): Jahresgutachten 2007/2008: Das Erreichte nicht verspielen, Stuttgart

Sesselmeier, W./Somaggio, G./Yollu, A. (2006): Mögliche Implikationen der gegenwärtigen Arbeitsmarktreformen für die zukünftige Entwicklung der Arbeitslosenversicherung, Studie im Auftrag der Hans-Böckler-Stiftung, Abschlussbericht, Landau

Storm, S./Naastepad, C. W. M. (2007): Why Labour Market Regulation May Pay off: Worker Motivation, Co-ordination and Productivity Growth, in: Economic and Labour Market Paper 2007/4, ILO 